\title{
Synthetic Doppler spectroscopy and curvilinear camera diagnostics in the ERO code
}

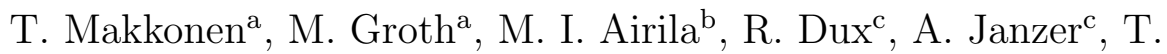 \\ Kurki-Suonio ${ }^{\mathrm{a}}$, T. Lunt ${ }^{\mathrm{c}}$, H.W. Mueller ${ }^{\mathrm{c}}$, T. Puetterich ${ }^{\mathrm{c}}$, E. Viezzer ${ }^{\mathrm{c}}$, \\ ASDEX Upgrade team \\ ${ }^{a}$ Aalto University School of Science and Technology, Association Euratom-Tekes, \\ FI-00076 AALTO, Finland \\ ${ }^{b}$ VTT Technical Research Centre of Finland, Association Euratom-Tekes, 02044 VTT, \\ Finland \\ ${ }^{c}$ Max-Planck-Institut für Plasmaphysik, EURATOM Association, Boltzmannstrasse 2, \\ 85748 Garching, Germany
}

\begin{abstract}
We present a set of new synthetic diagnostics, recently implemented in the ERO code, that were developed to facilitate direct comparisons between experiments and modelling of tokamak scrape-off-layer plasmas. The diagnostics calculate the spectroscopic Doppler shift and Doppler broadening of impurity lines of interest for any line of sight, as well as generate camera images from arbitrary viewing angles allowing for curvilinear (e.g. wide angle or fisheye) lenses. The synthetic camera diagnostics can either replicate the distortions caused by curvilinear lenses, or alternatively, create a rectilinear synthetic camera image and correct the curvilinear distortions in the experimental image. Comparison to experimental data is presented.
\end{abstract}

Keywords:

fusion, plasma, impurity, spectroscopy, camera, distortion, curvilinear

\section{Introduction}

Impurity transport in the scrape-off layer (SOL) of tokamaks is one of the extremely urgent issues because it is intimately tied to tritium retention, material migration, and impurity concentration in a fusion reactor. Numerical studies [1][2][3] have indicated that the SOL flow is one of the key factors in determining impurity transport. However, the SOL flow is poorly understood 
theoretically [4]. In order to gather more data on SOL flows [5], impurity injection experiments have been performed at ASDEX Upgrade (AUG) in a similar fashion as in $\mathrm{C}-\mathrm{Mod}$ [6][7]. In the AUG experiments, methane was injected from a valve located at the HFS about $13 \mathrm{~cm}$ above the midplane of the device. The ensuing CII $\left(\mathrm{C}^{1+}, 514 \mathrm{~nm}\right)$ and CIII $\left(\mathrm{C}^{2+}, 465 \mathrm{~nm}\right)$ emission distributions were followed with a spectrometer with 8 lines-of-sight [8] and two fast video cameras equipped with appropriate filters for CII and CIII. The spectrometer provides the Doppler shift of the carbon emission lines (the flow velocity) and the fast camera provides the shape of the emission distribution. The experimental setup is shown in figure 1. These experiments are reported in [9].

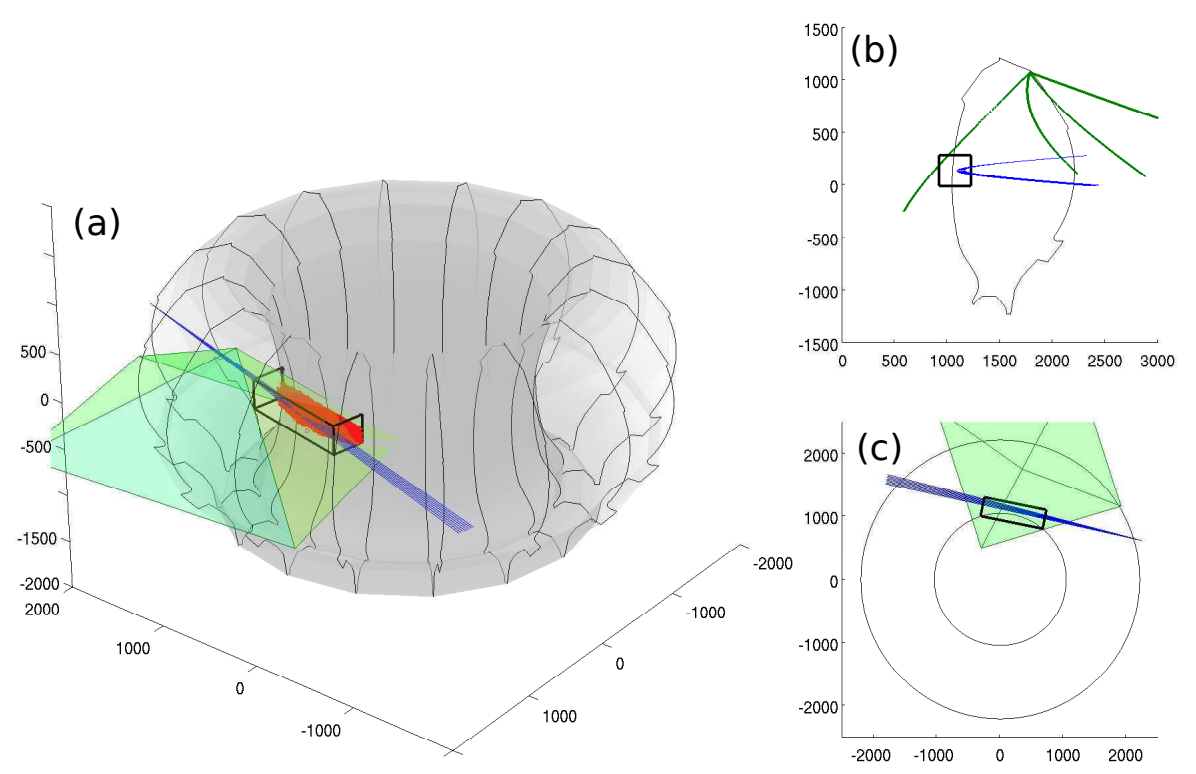

Figure 1: Experimental setup. (a) A 3D view of the AUG first wall, together with the ERO simulation box (drawn in thick black lines) and the appropriate diagnostics. The thin black lines and the grey surface represent the AUG first wall contours. The thin blue lines running trough the ERO simulation box are the spectroscopic lines-of-sight. The green translucent pyramid is the synthetic ERO fast camera view roughly corresponding to the real camera view. The red scattered data in the ERO simulation box is ERO simulation data. (b) A poloidal projection, (c) a horizontal projection as viewed from above.

It should be noted that the spectrometer observes a line-integrated signal. The macroscopic properties of the carbon plume (emission strength, flow velocity, etc.) change along the line-of-sight in these experiments. These are 
referred to as 3D effects in this paper. It is therefore important to understand spatial structure of the observed emission plume. The fast camera signal together with accurate reconstruction of the experiments can provide this.

The numerical reconstruction can be accomplished with the ERO code [10]. ERO is widely used to study impurity behavior in the SOL of fusion devices. It is a complex tool that, in a given background plasma and magnetic field, follows the trajectories of neutrals as well as charged ions and molecules. ERO includes a methane break up model and ionization of carbon as well as emissivity coefficients. However, the code does not include synthetic diagnostics that can directly be compared to the relevant AUG diagnostics. Therefore, a synthetic spectroscopy diagnostics and a synthetic camera diagnostic were implemented. These diagnostics were designed to replicate the diagnostics shown in figure 1 but are also easily transferred to another setup. This paper describes the new synthetic diagnostics, cites results where they are used for interpreting the experiment described above [9], and explores the importance of 3D effects for this particular case.

\section{Spectroscopic diagnostic}

The line integrated emission, the Doppler shift, and the Doppler broadening of the impurity emission can be calculated from the observed impurity spectra. The Doppler shift gives the flow velocity of the carbon ions, while their temperature can be deduced from the Doppler broadening of the lines. The new synthetic diagnostic does not reproduce the observed spectra but, instead, the line integrated emission, flow velocity, and thermal Doppler broadening for an arbitrary line-of-sight using emission data and various moments of the impurity velocity distribution. In the ERO code, this data is stored in a $3 \mathrm{D}$ grid.

The line integrated emission is trivially given by a line integral of the $3 \mathrm{D}$ emission data. To calculate the Doppler shift and Doppler broadening along a spectroscopic line of sight, the impurity velocity distribution function, as seen by the spectrometer line-of-sight, is required. The normalized velocity distribution is given as

$$
f_{\text {line }}(\vec{v})=\frac{\int_{\vec{x}} e(\vec{x}) f(\vec{x}, \vec{v}) d l}{\int_{\vec{x}} e(\vec{x}) d l}
$$

where the line integral is along the spectroscopic line of sight, $e(\vec{x})$ is the emission at position $\vec{x}$, and $f(\vec{x}, \vec{v})$ is the normalized distribution function. 
The Doppler shift, $u$, (here in units of velocity) along the spectroscopic line of sight is simply

$$
u=\int_{\vec{v}} f_{\text {line }}(\vec{v}) \vec{v} \cdot \vec{n} d \vec{v}=\frac{\int_{\vec{x}} e(\vec{x})\left(\int_{\vec{v}} f(\vec{x}, \vec{v}) v_{P} d \vec{v}\right) d l}{\int_{\vec{x}} e(\vec{x}) d l}
$$

where $v_{P}=\vec{v} \cdot \vec{n}$ is the velocity and $\vec{n}$ is the unit vector in the direction of the spectroscopic line of sight. The average velocity in the direction of the line-of-sight, i.e the flow velocity, at any given point is given by $\left\langle v_{P}\right\rangle=$ $\int_{\vec{v}} f(\vec{x}, \vec{v}) \vec{v} \cdot \vec{n} d \vec{v}$.

Similarly, the Doppler broadening (here in units of velocity squared) is given by

$$
T=\frac{\int_{\vec{x}} e(\vec{x})<\left(v_{P}\right)^{2}>d l}{\int_{\vec{x}} e(\vec{x}) d l}-u^{2}
$$

where $<\left(v_{P}\right)^{2}>$ is the average of the square of the velocity in the direction of the spectroscopic line-of-sight.

In order to allow for an arbitrary spectroscopic line-of-sight after the simulation has been finished, both $\left\langle v_{P}\right\rangle$ and $\left\langle\left(v_{P}\right)^{2}\right\rangle$ are calculated from quantities such as $\left\langle v_{x}\right\rangle$. The quantities $\left\langle v_{P}\right\rangle$ and $\left\langle\left(v_{P}\right)^{2}\right\rangle$ are given by

$$
<v_{P}>=<\vec{v} \cdot \vec{n}>=<v_{x}>n_{x}+<v_{y}>n_{y}+<v_{z}>n_{z}
$$

and

$$
\begin{aligned}
<v_{P}^{2}>= & <v_{x}^{2}>n_{x}{ }^{2}+<v_{y}{ }^{2}>n_{y}{ }^{2}+<v_{z}{ }^{2}>n_{z}{ }^{2} \\
& +2<v_{x} v_{y}>n_{x} n_{y}+2<v_{x} v_{z}>n_{x} n_{z}+2<v_{y} v_{z}>n_{y} n_{z} .
\end{aligned}
$$

In addition to the emission data, we thus need to keep track of the quantities $\left\langle v_{x}\right\rangle,\left\langle v_{y}\right\rangle,\left\langle v_{z}\right\rangle,\left\langle v_{x}^{2}\right\rangle,\left\langle v_{y}^{2}\right\rangle,\left\langle v_{z}^{2}\right\rangle,\left\langle v_{x} v_{y}\right\rangle,\left\langle v_{x} v_{z}\right\rangle$, and $\left\langle v_{y} v_{z}\right\rangle$. This can be greatly simplified by choosing the coordinate axes appropriately. For instance, for a spectroscopic line-of-sight with $n_{x}=1$, $n_{y}=0$, and $n_{z}=0$, it is only necessary to keep track of $\left\langle v_{x}\right\rangle$ and $\left\langle v_{x}^{2}\right\rangle$. The above process can naturally be extended to higher order terms were it necessary to know the features of the observed velocity distribution in greater detail.

It should be noted that there are other broadening mechanisms besides the thermal Doppler broadening. Broadening due to non-local effects are 
automatically taken into account in this method. This refers to situations where the macroscopic properties of the observed impurity plume change along the line-of-sight. For example, the observed Doppler broadening can be enhanced if the macroscopic flow velocity varies in different parts of the observed impurity plume. This is the case in the experiment presented in this work. However, other broadening mechanisms are not included. One such mechanism is turbulence [11]. The ERO code follows impurities in a smooth, steady-state plasma background and turbulence is not accounted for. Furthermore, the spectroscopy diagnostic presented here does not include effects such as pressure broadening or Stark broadening.

\section{Camera diagnostics}

The experimental setup presented in figure 1 utilized a wide angle lens producing curvilinear images. To properly study the shape of the carbon plume, these non-linear distortions have to be corrected for and the synthetic camera position and field of view correctly aligned.

In order to reconstruct the viewing geometry of the camera accurately, the AUG CAD models are represented within an OpenGL [12] 3D space, for which the same coordinate system is defined as for the real geometry. The virtual camera is placed at the position $\vec{c}$, corresponding to the real camera location. Into this same $3 \mathrm{D}$ space a semi transparent $2 \mathrm{D}$ mesh is plotted onto which the experimental camera image is mapped. Linear and non-linear transformations are applied to this mesh until the artificial AUG CAD models match with the experimental camera image. This is done using the augddd program [13] and illustrated in figure 2.

Using this process, it is possible to deduce from which direction the observed radiation in the image is coming from. In practice, this information is stored in a 2D grid linking positions in the image (e.g. position $(s, t)$ $=(0,0)$ referring to the lower left corner $)$ to a $3 \mathrm{D}$ vector, $\vec{d}$, pointing away from the camera. In the current implementation, the direction vectors are normalized so that they produce a plane as shown in figure 3 . This grid is referred to as the direction grid hereafter and is illustrated in figures 2 and 3 . The direction grid contains cells that are represented by four $(s, t)$ pairs and four corresponding vectors. The $(s, t)$ pairs are $\left(s_{a}, t_{a}\right),\left(s_{b}, t_{b}\right),\left(s_{c}, t_{c}\right)$, and $\left(s_{d}, t_{d}\right)$, where $a$ refers to bottom left, b refers to bottom right, c to top left, and $\mathrm{d}$ to top right. The grid is a square grid in $(s, t)$ space so that $s_{a}=s_{c}$, 


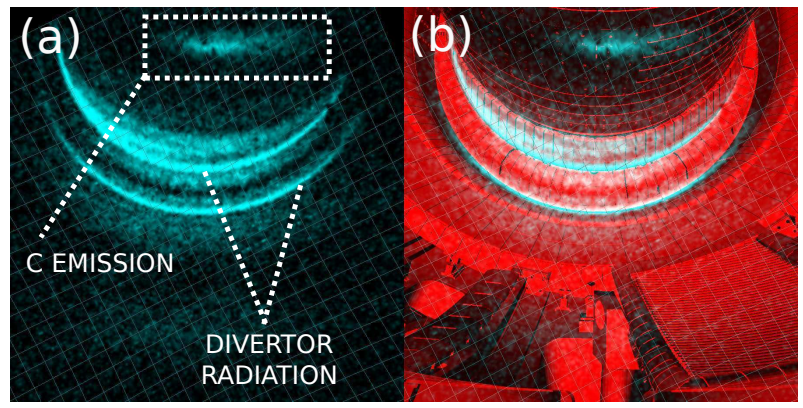

Figure 2: Two views from the augddd program. (a) Part of the experimental image. The injected carbon plume is visible at the top, and the divertor radiation in the middle. (b) The same image but translucent overplotted on AUG CAD drawings. Non-linear distortions have been applied to the image to try to match the AUG wall contours. The grid plotted on the image is the direction grid, visible in subfigure a, that is explained in the text.

$s_{b}=s_{d}, t_{a}=t_{b}$, and $t_{c}=t_{d}$. The corresponding vectors are $\vec{d}_{a}, \vec{d}_{b}, \vec{d}_{c}$, and $\overrightarrow{d_{d}}$.

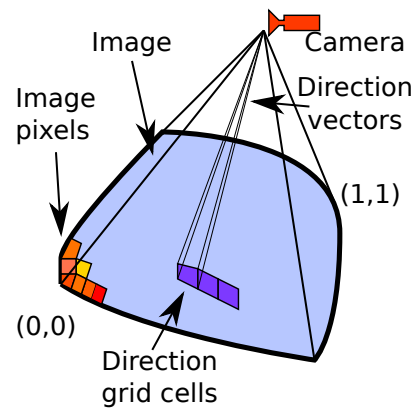

Figure 3: Visualization of a non-linear camera view cone with the direction grid described in the text. Three direction grid cells are drawn, along with the direction grid vectors for one cell. The position $(0,0)$ refers to the lower left corner of the image and $(1,1)$ refers to the top left corner of the image.

Two camera options were implemented in ERO. The first option is to create a simple, rectilinear, synthetic camera at the position of the real camera, looking roughly in the same direction as the real camera with roughly the same field of view. Using this option, the curvilinear distortions in the experimental image are corrected, and the experimental image is projected onto the view of the rectilinear synthetic camera.

Using the first option, every pixel in the experimental image is mapped 
to $3 \mathrm{D}$ space using the direction grid. This is visualized in figure 3. Every pixel in the experimental image has a corresponding $s$ and $t$ coordinate in the range $[0,1]$. Using these coordinates, the correct direction grid cell is identified to satisfy $s_{a} \leq s<s_{b}$ and $t_{a} \leq t<t_{c}$ The position inside the direction grid cell is given by

$$
u=\frac{s-s_{a}}{s_{b}-s_{a}}
$$

and

$$
v=\frac{t-t_{a}}{t_{c}-t_{a}}
$$

where $u$ and $v$ are in the range $[0,1]$. The position of the pixel in $3 \mathrm{D}$ space is then given by bi-linear interpolation as

$$
\vec{p}=\vec{c}+\left(\vec{d}_{a}(1-u)+\vec{d}_{b} u\right)(1-v)+\left(\vec{d}_{c}(1-u)+\vec{d}_{d} u\right) v .
$$

These pixels are then mapped to the view of the non-linear synthetic camera according to standard translation, rotations, and 3D projection operations. Similarly, the emission data given by ERO are represented in a 3D grid, which is essentially a set of points in 3D space. These points are mapped to the synthetic camera using the same projection operations. In this fashion, the corrected experimental image can be compared to the rectilinear synthetic camera.

It should be noted that using the first camera option, parts of the original experimental image can be lost (projected outside the view cone of the synthetic camera) and parts of corrected experimental image may contain no data.

In the second camera option, instead of using standard 3D projections to map the emission data given by ERO to a $2 \mathrm{D}$ image, equation

$$
\vec{p}=\vec{c}+\lambda\left(\left(\vec{d}_{a}(1-u)+\vec{d}_{b} u\right)(1-v)+\left(\vec{d}_{c}(1-u)+\vec{d}_{d} u\right) v\right)
$$

is solved for $\lambda, u$, and $v$. This is done for every point $(\vec{p})$ in the ERO emission data with respect to every direction grid cell $\left(\overrightarrow{d_{a}}, \overrightarrow{d_{b}}, \overrightarrow{d_{c}}\right.$, and $\left.\overrightarrow{d_{d}}\right)$. If the solution gives $u$ and $v$ in the range $[0,1]$ and a positive $\lambda$, we know the correct direction grid cell of the ERO emission data point. The corresponding $s$ and $t$ coordinates for the point $\vec{p}$ are given by bi-linear interpolation as $s=s_{a}(1-u)+s_{b} u$ and $t=t_{a}(1-v)+t_{c} v$, and the correct pixel in the synthetic image is determined by $s$ and $t$. 
The latter method is computationally expensive but has to be done only once after every ERO simulation. Yet another option to deal with non-linear distortions is to represent them using analytic formulas, but the method described here naturally allows for arbitrary distortions.

Either of these methods can be used to compare ERO simulations to experimental data. The first option has the advantage that straight lines remain straight. The second option has the advantage that the experimental data remains unmodified.

\section{Comparison to experiments}

As discussed in the introduction, ERO simulations with these synthetic diagnostics were used to interpret methane injection experiments in AUG [9]. Several similar discharges were carried out with methane injection in the HFS SOL. The filter for the camera was changed between discharges to record the ensuing CII and CIII emission profile. Also, the spectrometer wavelength was changed between discharges to observe the CII and CIII spectrum. The Zeeman structure of the emission was calculated using the magnetic field where the line-of-sight crosses the plane of injection to further deduce the Doppler shift (flow velocity) and Doppler broadening (temperature) from the observed spectra.

The flow of injected carbon provides a proxy for impurity transport but one would preferably try to access the background flow. ERO simulations were carried out for the setup shown in figure 1 for this reason. The aim was to match the experimental measurements by varying the plasma conditions in the HFS within a realistic range for density, temperature and flow velocity. The background plasma was assumed to depend only on the radial coordinate in the region of interest, close to the injection location, which is a reasonable assumption since gradients upstream are relatively small. Another assumption was that the parallel transport of the injected carbon is mainly determined by the parallel flow in the SOL.

ERO was able to reproduce the experimental measurements for lineintegrated emission and flow velocity for both CII and CIII using realistic values for the background [9]. However, the temperature was not matched. The comparison between experimental and ERO synthetic diagnostics is shown in figure 4 for the spectrometer. Figure 5 compares the signals from the experimental and synthetic fast camera diagnostics. This figure is generated 


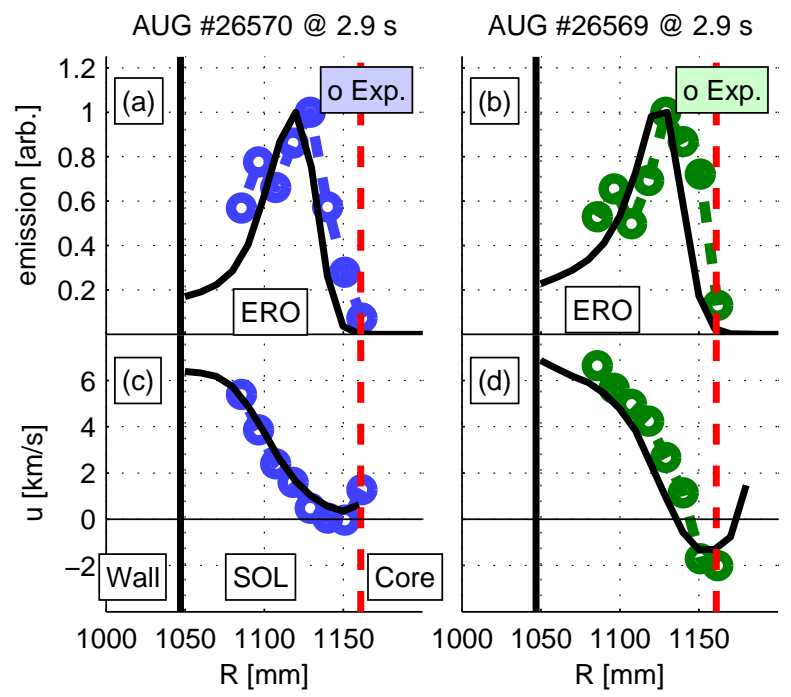

Figure 4: Comparison between synthetic ERO diagnostics (solid black line) and actual diagnostics for (a) line integrated emission for CII, (b) line integrated emission for CIII, (c) flow velocity of CII, and (d) flow velocity of CIII. The CII and CIII data was gathered during comparable discharges 26569 and 26570 while the ERO data is from from one simulation. The maximum of the line integrated emission is normalized to one as the amount of injected methane is not exactly known.

using the first, rectilinear camera option. The synthetic data in figures 4,5 , 6 , and 7 is from one ERO simulation.

The agreement between experimental and synthetic data is good, but it required careful optimization of the plasma profiles (density, temperature, and flow velocity) used in the ERO simulation [9]. Therefore, this cannot be considered a benchmark of the ERO code or the new diagnostics. The purpose was to use ERO to interpret the measurement withing the assumed model. This data is provided here as an example of the new diagnostics used in a realistic scenario. This ERO case is also used to study $3 \mathrm{D}$ effects.

As stated above, the Doppler broadening of CII and CIII was not matched with the observed temperature being higher than given by the ERO code. The observed temperature for CIII in ERO is in the range of $5 \mathrm{eV}$ while the experimental data is in the range of $15 \mathrm{eV}$. Broadening due to the Zeeman effect has been taken into account on the experimental side when analyzing the observed CII and CIII spectra. Besides the Zeeman effect and the thermal 

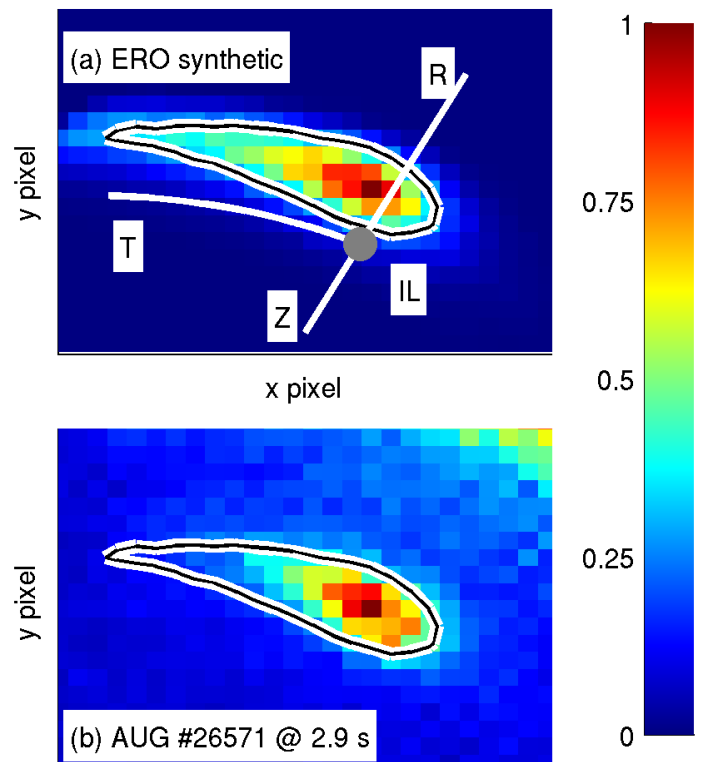

Figure 5: Comparison between (a) synthetic ERO camera and (b) actual camera image for CII emission. This figure is generated using the rectilinear camera option 1 . The maximum of emission os normalized to one as the amount of injected methane is not known. TL indicates the injection location. $\mathrm{R}$ indicates a radial vector starting from the injection location extending $300 \mathrm{~mm}$ towards the core of the plasma. $\mathrm{Z}$ is is $150 \mathrm{~mm}$ long $\mathrm{z}$-directional vector. $\mathrm{T}$ indicates a $20^{\circ}$ degree long $(\approx 365 \mathrm{~mm})$ circle arc starting from the injection location going in the toroidal direction. This is approximately the direction of the magnetic field and the background flow.

Doppler broadening, no other broadening mechanism are included. This is potentially a reason for the large discrepancy. Another is potentially missing physics in the simulations. Work to investigate this discrepancy is ongoing. Possible other mechanism include turbulence and Stark broadening.

Despite the temperature discrepancy, it is assumed that the shown ERO reconstruction is realistic enough to be used to study the importance of $3 \mathrm{D}$ effects. A simple test is the level of convolution for the spectrometer linesof-sight. Figure 6 shows the line-integrated emission profile calculated by the synthetic ERO diagnostic compared to the flux surface averaged radial emission profile from the ERO simulations. As it is assumed that the ERO simulations represent the actual experiment, figure 6 essentially represents a deconvolution of the experimentally observed data. From figure 6 it can be seen that 3D effects are important for the outermost lines-of-sight (close 


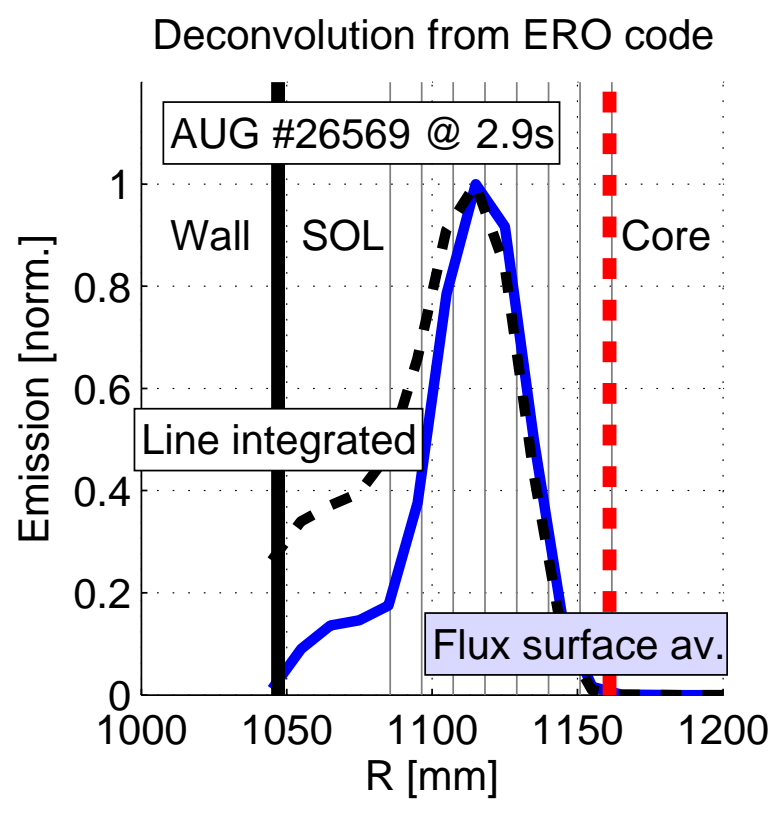

Figure 6: Comparison between the radial CII emission profile produced by the synthetic ERO diagnostic (dashed black line) and the flux surface averaged radial emission profile in the ERO simulations (solid blue line). The vertical thin grey lines indicate the 8 spectroscopic lines-of-sight.

to the wall) where the line-integrated emission strength is larger than the toroidally averaged emission strength. This is because the outermost linesof-sight partially observe the carbon plume going around the central column. For the innermost lines-of-sight, 3D effects appear negligible.

The shown ERO reconstruction is also used in this paper to display the difference with the two camera options. Figure 7 displays ERO camera images generated with camera options 1 (rectilinear) and 2 (curvilinear) together with the innermost and outermost spectroscopic lines-of-sight. With the rectilinear camera option, straight lines remain straight.

\section{Summary and conclusions}

To facilitate the analysis of impurity injection experiments, new synthetic diagnostics have been implemented into the ERO code. A synthetic spectroscopy diagnostic that can calculate the line integrated emission, the Doppler shift, and Doppler broadening along an arbitrary line of sight is now included in ERO. In addition, a synthetic camera diagnostic that allows any 

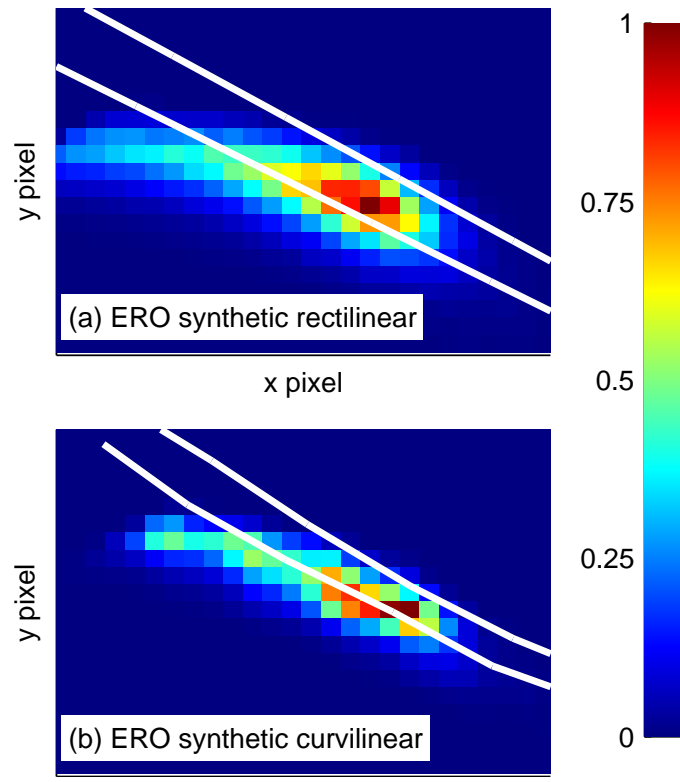

0.25

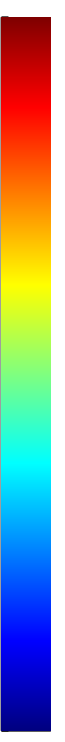

Figure 7: Comparison between camera options 1 and 2 for the ERO synthetic camera for CII emission. The thin white lines indicate the innermost (lower) and outermost (higher) spectroscopic lines-of-sight.

viewing angle and location has been implemented. This synthetic camera diagnostic can either replicate the curvilinear distortions seen in the experimental image, or correct the curvilinear distortions from the experimental image and compare it to a rectilinear synthetic camera image. Careful simulations of the experiments together with these diagnostics provide now the means for accessing the parameters determining the transport of carbon, specifically the background flow.

The importance of 3D effects can vary depending on the plasma conditions and the used impurity species. Also, the curvilinear distortions of the optical system vary from system to system. The synthetic diagnostics and method presented here will, however, allow to easily study an arbitrary case in a systematic fashion.

\section{Acknowledgments}

The ERO code is maintained by Forschungszentrum Jülich, Institut für Energie- und Klimaforschung Plasmaphysik. This work, supported by the 
European Communities under the contract of Association between EURATOM/Tekes, was carried out within the framework of the Task Force on Plasma Wall Interactions of the European Fusion Development Agreement. The views and opinions expressed herein do not necessarily reflect those of the European Commission. This work was funded by Academy of Finland project numbers SA-253222 and SA-134924

\section{References}

[1] Strachan J.D. et al Nucl. Fusion 44 (2004) 772

[2] McLean A.G. et al J. Nucl. Mater 337-339 (2005) 124

[3] Elder J.D. et al. J. Nucl. Mater. 363-365 (2007) 140

[4] Groth M. et al Nucl. Fusion 49 (2009) 115002

[5] Asakura N. et al. J. Nucl. Mater. 363-365 (2007) 41

[6] Gangadhara S. et al J. Nucl. Mater. 290-293 (2001) 598

[7] Gangadhara S. et al J. Nucl. Mater. 313-316 (2003) 1167

[8] Puetterich T. el al Nucl. Fusion 52 (2012) 083013

[9] Makkonen T et al. J. Nucl. Mater. (2013) in press http://dx.doi.org/10.1016/j.jnucmat.2013.01.082

[10] Kirschner A. et al. Nucl. Fusion 40 (2000) 989

[11] H.R. Griem, Principles of plasma spectroscopy, Cambridge University Press (2005)

[12] Open graphics library (OpenGL) http://www.opengl.org/

[13] Lunt T. et al. Nuclear Instruments and Methods in Physics Research Section A: Accelerators, Spectrometers, Detectors 623 (2010) 812 